EPJ Web of Conferences 41, 07012 (2013)

DOI: $10.1051 /$ epjconf/20134107012

(C) Owned by the authors, published by EDP Sciences, 2013

\title{
Optimal Dynamic Discrimination in Tryptophan-Containing Dipeptides
}

\author{
S. Afonina, O. Nenadl, A. Rondi, D. Kiselev, J. Extermann, L. Bonacina and J.-P. Wolf \\ GAP - Biophotonics, University of Geneva, Geneva, Switzerland
}

\begin{abstract}
Optimal Dynamic Discrimination based on the phase-shaping of deep ultraviolet femtosecond pulses was applied to selectively modulate the time-resolved fluorescence depletion of pairs of tryptophan-containing dipeptides. Our results indicate that phase-sensitive excitation allows their differential identification, beyond the limits of linear and time-resolved spectroscopy.
\end{abstract}

\section{Introduction}

The essential amino acid tryptophan (trp) and its dipeptide counterparts alanine- (alatrp), glycine- (gly-trp), and leucine-tryptophan (leu-trp) are ubiquitous molecules of high biological significance. In particular, trp is the main probe for UV-vis spectroscopy, thanks to its high fluorescence quantum yield and large Stokes shift. The high sensitivity of trp to the environment is well known, and, in this respect, trp bound to a single amino-acid by a peptide bond represents a perfect workbench for research.

The spectroscopic properties of all the molecules under scrutiny are dominated by those of trp, and, in fact, they present identical absorption and strongly overlapping florescence spectra, making their discrimination by linear spectroscopy nearly impossible (Fig. 1a). Interestingly, even time-resolved approaches (i.e. fluorescence depletion), which proved successful in the past for discriminating trp-containing samples [1], are not selective enough in the present case. On the other hand, our group has demonstrated how, within the framework of Optimal Dynamic Discrimination (ODD) [2], a careful manipulation of pulse spectral-phase can drive the selective excitation of the low-frequency molecular modes in spectroscopycally identical biochromophores, eventually leading to their fluorescence-based identification in a mixture [2,3]. Moreover this method has shown high discrimination power in the fluorescence depletion experiment for two essential aromatic amino acids: tryptophan and tyrosine. By use of acousto-optic programmable dispersive filter (AOPDF), commercially known as Dazzler (Fastlite), fluorescence modulation was achieved with a contrast of 35\% [4].

In this contribution, we demonstrate that, by employing a new dedicated reflective shaper [5], the ODD approach can be readily extended from the UV-visible to the deep UV spectral region, accessing the absorption bands of amino-acids, peptides, and possibly DNA. 


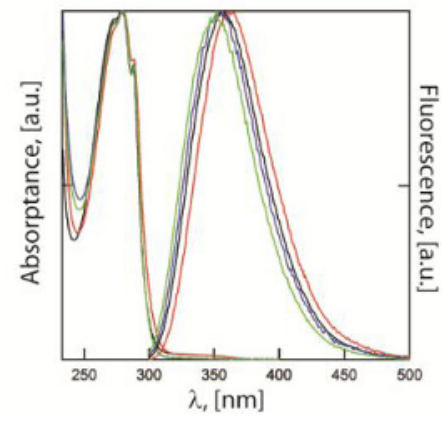

a)

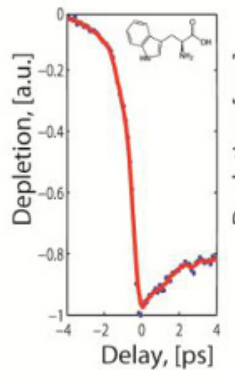

b)

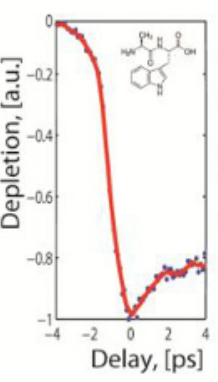

c)

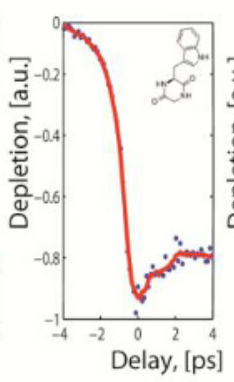

d)

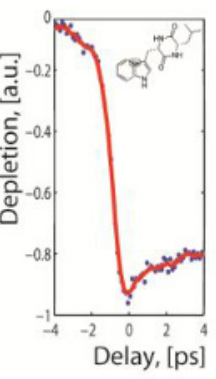

e)

Fig. 1a. Normalized absorption and fluorescence spectra, same molar concentrations, for the four molecules, showing a near perfect overlap. Black: trp, red: ala-trp, blue: cyclo(-gly-trp), green: cyclo(leu-trp).

Fig. 1a-e. Normalized fluorescence depletion curves for tryptophan (b), alanine-trp (c), cyclo(-gly-trp) (d) and cyclo(-leu-trp) (e) obtained by Fourier-Transform limited pulses. The relevant molecular structures are also shown.

\section{Results and Discussion}

As one can see from Fig. 1, the fluorescence depletion transients measured on trp and the three dipeptides under study are indistinguishable when close-to-Fourier-Transform pulses are employed for excitation $(266 \mathrm{~nm})$ and depletion $(800 \mathrm{~nm})$. To counteract this lack of selectivity, we ran a series of feedback driven optimizations aimed at enhancing the difference in the fluorescence depletion response yielded by pairs of molecules interrogated simultaneously. Thus the goal was to maximize or minimize the quantity of depletion value with respect to the reference fluorescence depletion with unshaped pulse.

Example of successful optimization experiment for trp and ala-trp one can see on the fig. 2a-c. More specifically, the spectral phase of the UV pulse was modified according to the evolution of a multi-objective genetic algorithm [6]. Depletion ratio was obtained from measurement sets (each set is the average of 1000 measurements) for generating statistically relevant figures.

For several molecule pairs (trp vs ala-trp, trp vs cyclo(-gly-trp), cyclo(-gly-trp) vs ala-trp) the discrimination capability of the approach is the order of $20 \%$, which is remarkably high considering the modest bandwidth available at $266 \mathrm{~nm}(3 \mathrm{~nm}$ FWHM). This restricted parameter space does not allow, in opposite, to discriminate other dipeptides like ala-trp and cyclo(-leu-trp). 


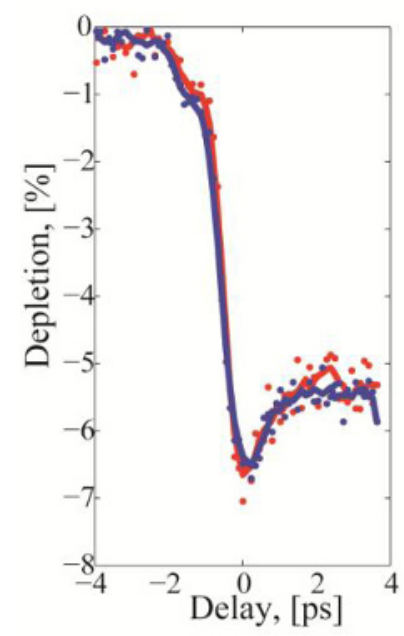

a)

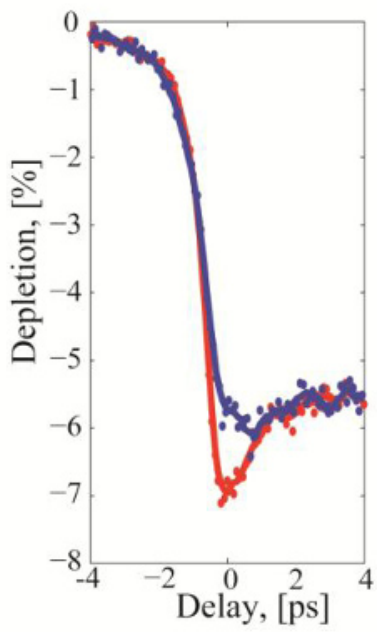

b)

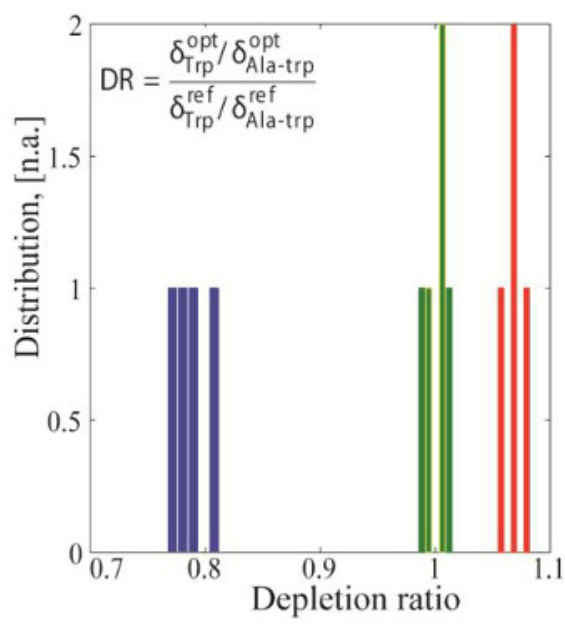

c)

Fig. 2a-b. Example of successful optimization for fluorescence depletion modulation in case of trp (a) and ala$\operatorname{trp}(\mathrm{b})$.

Fig. 2c. Histogram of fluorescence depletions for trp and ala-trp. Green (middle column): reference obtained with Fourier-Transform pulses, Blue (left column): maximization of fluorescence depletion for trp, Red (right column): maximization of fluorescence depletion for ala-trp.

\section{Conclusions}

We showed the phase-sensitive modulation of the fluorescence depletion of trp and trp-containing dipeptides with the aim of fluorescence-based identification of otherwise spectrally indistinguishable molecules. This work demonstrates that ODD can be extended to the manipulation of optical response of deep UV absorbing biochromophor like proteins and even DNA.

\section{References}

1. F. Courvoisier, L. Bonacina, V. Boutou, L. Guyon, C. Bonnet, B. Thuillier, J. Extermann, M. Roth, h. Rabitz, J.-P. Wolf, Faraday Discussions 137, 37-49 (2008).

2. M. Roth, L. Guyon, J. Roslund, V. Boutou, F. Courvoisier, J.-P. Wolf, H. Rabitz, Phys. Rev. Lett. 102, 253001 (2009)

3. J. Petersen, R. Mitric, V. Bonacic-Koutecky, J.-P. Wolf, J. Roslund, H. Rabitz, Phys. Rev. Lett. 105, 073003 (2010)

4. S.-M. Weber, L. Bonacina, W. Noell, D. Kiselev, J. Extermann, F. Jutzi, S. Lani, O. Nenadl, J.P. Wolf, N.-F. de Rooij, Rev. Sci. Instrum. 82, 075106 (2011)

5. A. Rondi, L. Bonacina, A. Trisorio, C. Hauri, J.-P. Wolf, Phys. Chem. Chem. Phys.14, 9317$9322(2012)$

6. L. Bonacina, J. Extermann, A. Rondi,V. Boutou, J.-P. Wolf, Phys. Rev. A. 76, 023408 (2007) 